\title{
Probiotics for Everyone! The Novel Immunobiotic Lactobacillus rhamnosus CRL1505 and the Beginning of Social Probiotic Programs in Argentina
}

\author{
Julio Villena ${ }^{1}$, Susana Salva1, Martha Núñez ${ }^{2}$, Josefina Corzo ${ }^{3}$, René Tolaba ${ }^{4,5}$, \\ Julio Faedda ${ }^{4}$, Graciela Font ${ }^{6}$ and Susana Alvarez ${ }^{1,7}$,
}

${ }^{1}$ Laboratory of Clinical and Experimental Biochemistry, Reference Centre for Lactobacilli (CERELA-CCTCONICET), Chacabuco 145 - T4000ILC, San Miguel de Tucumán, Tucumán, Argentina

${ }^{2}$ Laboratory of Experimental Foods, Reference Centre for Lactobacilli (CERELA-CCT-CONICET), Argentina

${ }^{3}$ Nutrition Division, Integrated Health Programs, Ministry of Public Health, Government of Tucuman, San Lorenzo 1142, CP4000, San Miguel de Tucumán, Tucumán, Argentina

${ }^{4}$ Del Niño Jesus Children Hospital, Pasaje Hungría 750, CP4000, San Miguel de Tucumán, Tucumán, Argentina

${ }^{5}$ Department of Child and Adolescent Medicine, Faculty of Medicine, National University of Tucuman, Ayacucho 471, CP4000, San Miguel de Tucumán, Tucumán, Argentina

${ }^{6}$ Laboratory of Technology and Development, Reference Centre for Lactobacilli (CERELA-CCT-CONICET), Argentina

${ }^{7}$ Institute of Applied Biochemistry, Faculty of Biochemistry, Chemistry and Pharmacy, National University of Tucuman, Balcarce 747, CP4000, San Miguel de Tucumán, Tucumán, Argentina

\begin{abstract}
Lactobacillus rhamnosus CRL1505 (Lr1505) stimulates immune responses in the gut and in the respiratory tract and improves resistance against Salmonella typhimurium and Streptococcus pneumoniae infections in immunocompetent and immunocompromised mice. Considering that respiratory infectious diseases continue to be a major cause of death among preschool children in developing countries, the aim of the present study was to evaluate the effect of Lr1505 on the health of children. A randomized-controlled double-blind clinical trial in 298 healthy children (2-5 years old), attending daycare centers was performed. Yogurt containing Lr1505 was administered to children for 6 months (five times a week). Results were statistically compared with those of children from the same community that received a placebo yogurt (without probiotic). Administration of Lr1505 to young children reduced the incidence of infections: $66 \%$ of children in the placebo group presented symptoms of infection while only $34 \%$ of cases were detected in the Lr1505 group. Significant differences $(P<0.05)$ were detected in the incidence of intestinal infections, upper respiratory tract infections and angina when placebo and Lr1505 groups were compared. Children fed Lr1505 experienced fewer fevers and needed fewer antibiotics than those receiving the placebo. The protective effect of Lr1505 was associated with increased levels of mucosal IgA antibodies. Lr1505 is a promising resource for the development of prevention strategies against mucosal infections that could be effective tools for medical application. This new probiotic strain has been included into official Nutritional Programs in Argentina and it is given to more than 200 thousand children. This project has encouraged local milk production, thanks to the constant demand of probiotic yogurt containing L. rhamnosus CRL1505 by provincial governments, while incorporating innovation to small and medium enterprises.
\end{abstract}

Keywords: Lactobacillus rhamnosus CRL1505, children, mucosal immunity, respiratory infections.

\section{INTRODUCTION}

Common infectious diseases continue to be a major cause of death among preschool children in developing countries [1-3]. The increase in antibiotic resistance and need for new and improved strategies to tackle infectious disease have led to an examination of the therapeutic potential of commensal induced modulation of the mucosal immune response. Consequently, it has been discovered that certain probiotic lactic acid

*Address corresponding to this author at the Laboratory of Clinical and Experimental Biochemistry, Reference Centre for Lactobacilli (CERELACONICET), Tucuman, Argentina; Tel: 54-381-4310465; Fax: 54-381-4005600; E-mail: salvarez@cerela.org.ar bacteria (LAB) do have protective effects against bacterial and viral infections in the gastrointestinal tract [4]. Significant attention has been focused on the role of probiotics in the protection of gut against pathogens. However, there is steadily increasing evidence that orally delivered probiotics are able to regulate immune responses outside the gastrointestinal tract, including the respiratory mucosa [5]. In mouse studies, it has been demonstrated that oral administration of probiotic $L A B$ protects against respiratory pathogens such as Streptococcus pneumoniae [6, 7], Pseudomonas aeruginosa [8] and influenza virus [9]. Moreover, some human trials demonstrated that administration of probiotics has been associated with lower incidence of 
ventilator-associated pneumonia [10], reduced respiratory infections in healthy and hospitalized children [11-13] and reduced duration of common cold infection [14].

With the aim to develop a new functional food able to improve both intestinal and respiratory immunity, we have evaluated the immunomodulatory capacity of several lactobacilli strains isolated from goat milk and we found that Lactobacillus rhamnosus CRL1505 stimulates the innate and adaptive immune response in the gut in a dose-dependent way and confers resistance to infection with Salmonella typhimurium in immunocompetent [15] and immunocompromised malnourished [16] mice. Moreover, we have demonstrated that orally administered L. rhamnosus CRL1505 is able to increase the resistance to pneumococcal infection in immunocompetent mice and that this effect is associated with improvements of innate and adaptive immune responses in the respiratory tract [15]. In addition, we recently demonstrated that the use of $L$. rhamnosus CRL1505 as a supplement in a repletion diet is able to improve the number and functionality of immune cells that participate in the response against a pneumococcal infection in malnourished mice [16].

Our studies in mice provided clear evidence that $L$. rhamnosus CRL1505 improves immune responses in the gut and beyond the gastrointestinal tract. However, the immunomodulatory effect of this strain was not tested in humans before. Therefore, in the present work we aimed to evaluate the effects of a probiotic yogurt containing the strain L. rhamnosus CRL1505 on the health of children attending pre-school daycare community centers. We evaluated the impact of probiotic yogurt containing $L$. rhamnosus CRL1505 on mucosal immunity and study its effect on the incidence and severity of gastrointestinal and respiratory infections.

\section{MATERIALS AND METHODS}

\section{Study Design}

A randomised, double-blind, placebo-controlled study was conducted to evaluate the effect of the consumption of a probiotic yogurt on health of children. One children group was randomly allocated to the probiotic yogurt and the other to a placebo product. The study consisted of 6 months product consumption between July and December. The trial was conducted by nutritionists (SIPROSA), pediatricians (Tucuman
Children Hospital), and immunologists (CERELA). In addition, daycare center staff was previously trained and allowed to participate. The experimental protocol and informed consent for parents were previously approved by the Ethics Committee of the Faculty of Medicine of the Tucuman University (Res. № 1121-06).

\section{Participants}

Healthy children attending daycare centers fivedays a week in Tucuman (Argentina) were recruited into the study. The purpose of the study was explained to the parents and a written informed consent was obtained. Procedure consisted of supervisor visiting the household and in presence of a third party, obtaining the consent from the mother or father after reading the consent form to them.

Our inclusion criteria were: children between 2 and 5 years old, from both sexes, adequate nutrition, attending daycares, who voluntarily agree to participate in the study, with written consent of parents or guardians. Before inclusion in the study, participants and the adults responsible for children were informed about the nature and characteristics of the study. Only those who met the eligibility criteria and whose parents or guardians gave their written consent to participate were included in the trial.

Exclusion criteria were: congenital disease affecting the digestive system (cystic fibrosis, celiac disease, allergy to cow's milk), chronic diseases (pulmonary dysplasia, COPD, chronic renal failure). In addition, children or parents which do not accept participating in the study were not included.

\section{Intervention}

Children were randomised to receive either the probiotic yogurt or the placebo product. The probiotic yogurt was sweetened, flavoured, fermented milk containing at least $10^{8}$ colony-forming units (CFU)/100 $\mathrm{g}$ of the probiotic strain Lactobacillus rhamnosus CRL1505 with a starter culture for yogurt prepared with Streptococcus thermophilus and Lactobacillus delbrueckii subsp. bulgaricus strains from the collection culture of CERELA. L. rhamnosus CRL1505, isolated from goat milk from northwestern Argentina, was previously selected by its ability to stimulate the immune system by increasing resistance to infection in the respiratory and intestinal tracts [15-17]. The placebo product was fermented milk produced with same starter culture without L. rhamnosus CRL1505. 
The nutritional composition, appearance, taste and packaging of the probiotic yogurt and the placebo were identical throughout the study in order to maintain blinding. Enrolled children were randomly allocated to the two intervention groups and the participants were masked to group allocation. In addition, all the persons involved in the study, the different health professionals as well as the parents and guardians were unaware of the randomization codes. The secret codes were secured until completion of data entry for analysis.

The Laboratory for Experimental Foods (CERELACONICET) prepared the yogurt and probiotic cultures. The final products were elaborated by the dairy company "Al Pie de la Vaca" and were cold-stored $\left(8^{\circ} \mathrm{C}\right)$ in daycares.

The probiotic yogurt and the placebo were administered daily from Monday to Friday (five days per week). During the study, children had to ingest one bottle of $100 \mathrm{~g} /$ day of yogurt or placebo product.

Before initiation of the study, children underwent a clinical examination including anthropometric measures (weight, height). In addition, children and parents underwent a clinical nutrition questionnaire (which included personal data, medical history, familyinherited diseases, psychomotor, environmental and sociocultural skills).

The children were randomly allocated to probiotic yogurt or the placebo group on day zero of the study. Total of 298 children participated and were divided in two groups: 150 children were administered the probiotic yogurt while 148 children received the placebo. The clinical and nutritional monitoring was conducted by pediatricians from the Tucuman Children
Hospital and by nutritionists from the SIPROSA Nutrition Division (Tucuman Government).

During the study, visual controls of both placebo and probiotic yogurt consumption were performed by nutritionists. It was recorded that $100 \%$ of the products -probiotic yogurt and placebo- were consumed by the participants throughout the studied period.

\section{Assessment of Product Acceptability Using Hedonic Scale}

We assessed the quality of the product from the subjective point of view, considering that the success or failure of a food product depends not only on its composition or nutritional value, but also on subjective reactions associated with a pleasurable sensation. A hedonic test for children was used. The scale used was the hedonic faces with different facial expressions ranging from anger (complete dissatisfaction) to happy (complete satisfaction). Children expressed their opinion concerning a pleasant character on this scale. Yogurt and placebo products were coded so that recipients could not identify the product. The assessment of overall satisfaction was greater than $90 \%$ in the strawberry- and vanilla-flavored yogurt.

\section{Follow Up Observation}

Planed visits were conducted once a week by pediatricians. In these visits, clinical examination of each child was performed as well as nutritional assessment (weight/age, height/age and weight/height ratios), according to the standards of the Provincial Health System of Tucumán (SIPROSA).

In addition, during the trial the occurrence of infectious events related to the gastrointestinal and

Table 1.

\begin{tabular}{|c|c|c|}
\hline Infection & Main causative pathogen in children under 5 years & Reference \\
\hline \hline Acute otitis media & Streptococcus pneumoniae and Haemophilus influenzae & [18] \\
\hline $\begin{array}{c}\text { Pharyngitis and tonsillitis } \\
\text { Upper airway infections } \\
\text { (common cold) }\end{array}$ & $\begin{array}{c}\text { Respiratory syncytial virus, Human metapneumovirus, Influenza A virus, } \\
\text { Parainfluenza viruses and Rhinoviruses }\end{array}$ & [20] \\
\hline Pneumonia & $\begin{array}{c}\text { Streptococcus pneumoniae, Haemophilus influenzae and Mycoplasma } \\
\text { pneumoniae }\end{array}$ & [21] \\
\hline Bronchitis & Respiratory syncytial virus, Parainfluenza viruses and Rhinoviruses & [22] \\
\hline Diarrhea & Rotavirus and Adenovirus & [23] \\
\hline $\begin{array}{c}\text { Skin infections } \\
\text { (impetigo and pyodermitis) }\end{array}$ & Staphylococcus aureus and $\beta$-hemolytic streptococci \\
\hline
\end{tabular}


respiratory systems was recorded. Data collection was performed by a survey performed by paediatricians. The data were entered in individual forms for each child where the date and infectious characteristics were registered. The most common infectious diseases in children under five years were analysed: Acute otitis media, pharyngitis and tonsillitis, upper airway infections (common cold), pneumonia, bronchitis, diarrhoea and skin infections (impetigo and pyodermitis) (Table 1).

\section{Laboratory Tests}

Samples of faeces were collected at the beginning and end of the study in order to determine lactic acid bacteria counts.

In addition, levels of IgA in saliva were determined. Samples were taken the day before the beginning of probiotic yogurt or placebo administration (Basal levels) and at the end of their administration (Post treatment levels). Saliva samples were collected from 200 children (100 yogurt group and 100 in the placebo group) with sterile pasteur pipette and placed in appropriately labeled eppendorf tubes. Immediately after collection, the samples were cooled for transport to the laboratory. The samples were clarified by centrifugation at $2,500 \mathrm{~g}$ for 10 minutes, separated into aliquots and stored at $-70 \circ \mathrm{C}$ until antibody assessment. Measurements were performed using the ELISA technique using anti-human IgA (Sigma \# 10884). Total levels of antibody were quantified by linear regression analysis of the values of optical density (OD) of samples against a standard curve consisting of 10 serial dilutions of human IgA (Sigma \# S5018). All the analytical dosages in the study were performed in blind.

\section{Data Analysis}

Statistics Division from CERELA was responsible for analysis of results. StatsDirect software was used. Appropriate parametric and non-parametric methods were employed according to the distribution of the data. The Student $t$ test was used to compare means of the continuous variables with normal distribution. For variables with no normal distribution, the Mann-Whitney $\mathrm{U}$ test was used. The $\mathrm{X} 2$ test or Fisher exact test were used to compare percentages. The confidence interval of $95 \%$ was calculated using the same software. The difference between groups was considered significant for $p<0.05$.

\section{RESULTS}

\section{Incidence of Infections}

As shown in Figure 1, there were 298 children enrolled in the study; 150 received the probiotic yogurt containing L. rhamnosus CRL1505 and 148 received the placebo product. There was no statistically significant difference between the groups in regard to age, gender, weight and height at the beginning of the study, or difference in weight and height prior to and after the intervention. Of the total of 298 children who participated, $132(45 \%)$ of them presented infectious events during the trial (Figure 2A). The most common infectious diseases were upper respiratory infections followed by pharyngitis and tonsillitis, lower respiratory infections (acute bronchitis) and diarrhea (Figure 2A). In the group of children that recieved the yogurt containig the probiotic strain L. rhamnosus CRL1505 it was observed a significant decrease of infectious events when compared to the placebo group (34\% vs $66 \%$ ) (Figure 2B). When the different types of infectious diseases were analyzed, we observed significant differences between the yogurt and placebo groups in the following events: upper respiratory infections ( $31 \%$ vs $69 \%)$, pharyngitis and tonsillitis ( $28 \%$ vs $72 \%)$ and acute diarrhea (26\% vs $74 \%$ ). No differences were observed in the incidence of bronchitis (Figure 2B). In addition, the number of children suffering pneumonia, otitis media or skin infections (impetigo and pyodermitis) did not allow us to perform statistical analysis (Figure 2B). Symptom duration of infection events for subjects in the placebo and the probiotic yogurt groups were not different (Figure 3A). The clinical effects of probiotic supplementation on the incidence of fever and the need of antibiotic tretament were also evaluated. Subjects in the probiotic yogurt group were found to have significantly lower incidence of having fever (Figure 3B). Furthermore, the need of antibiotic treatment among the probiotic-consuming children was significantly lower than that in the placebo group (Figure 3C).

\section{Lactic Acid Bacteria in Stools}

Analysis of the amount of LAB species in stools was performed after 6 months of product consumption. Comparison between groups was analysed by using a Mann-Whitney test. The results show no statistical difference between groups in the amount of $L A B$ species in stools ( $P=0.6017$; Figure 4$)$. 


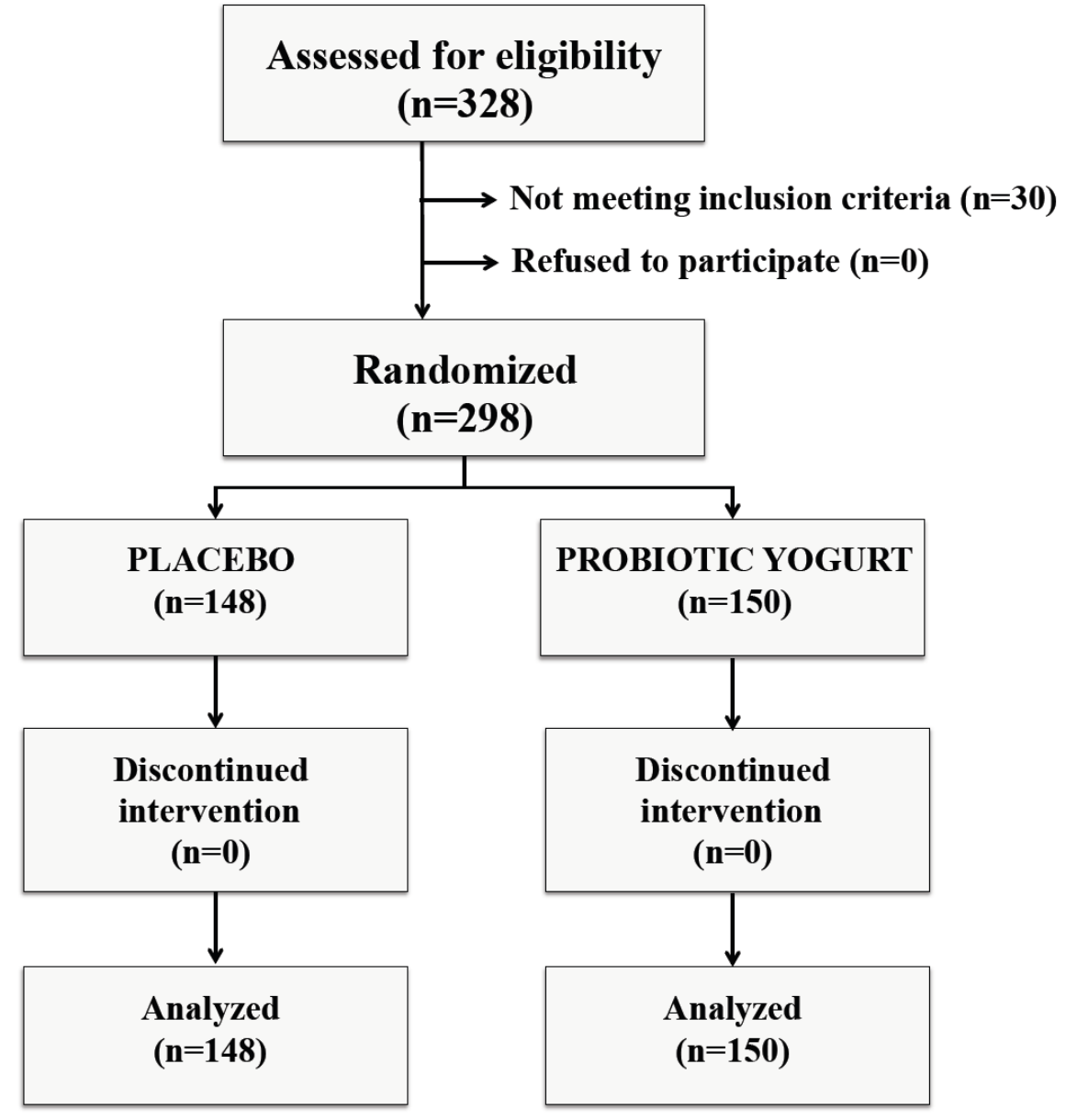

Figure 1: Diagram showing the flow of participants through the stages of the study.

A

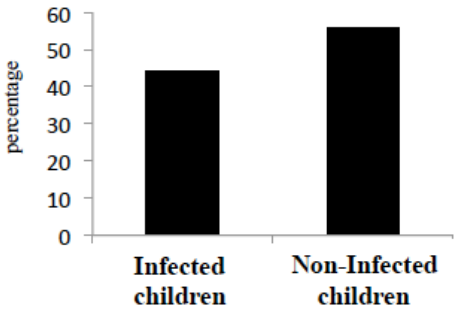

C

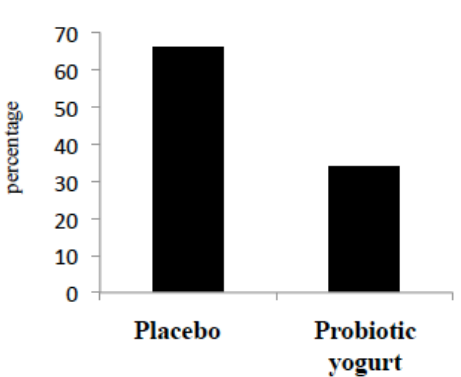

B

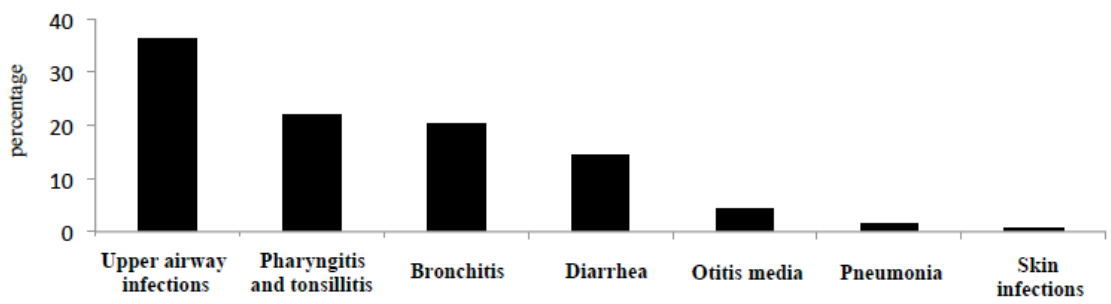

D

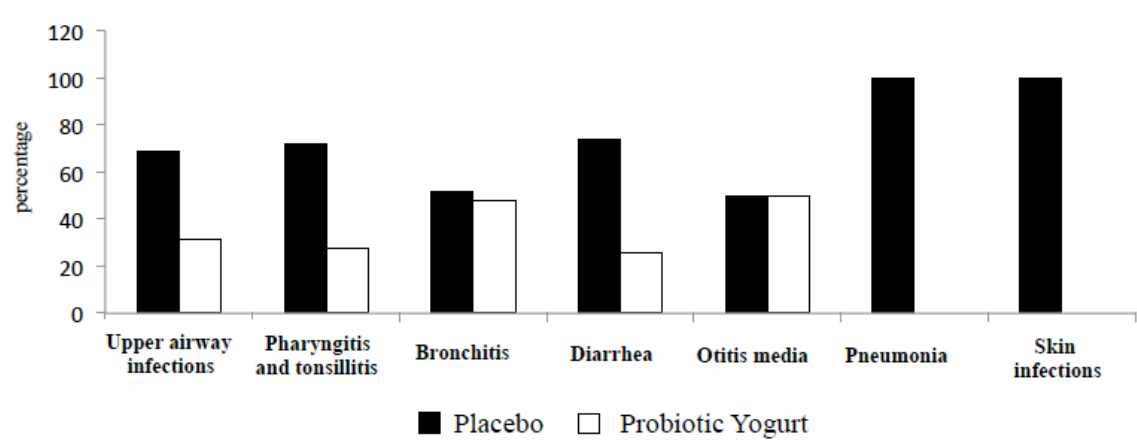

Figure 2: Effects of a probiotic yogurt containing the strain Lactobacillus rhamnosus CRL1505 on the incidence of common infectious diseases in pre-school children. (A) Incidence of infections in the group of children, (B) different types of infectious diseases. (C, D) Effect of probiotic yogurt consumption in the incidence of infections. 


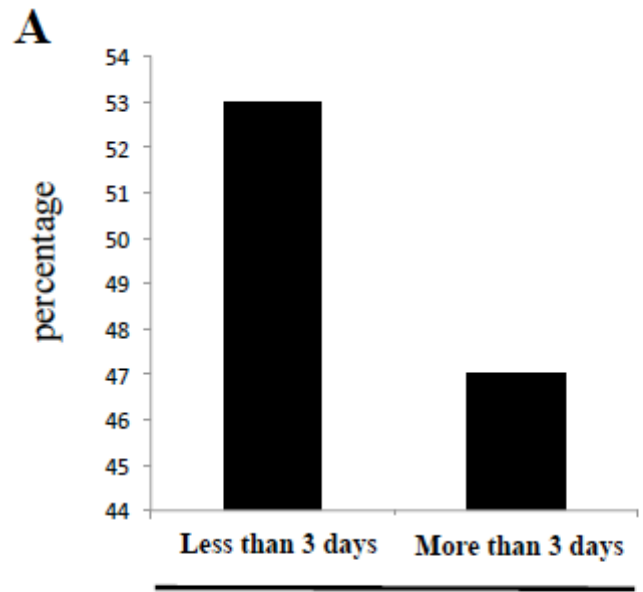

Placebo

B

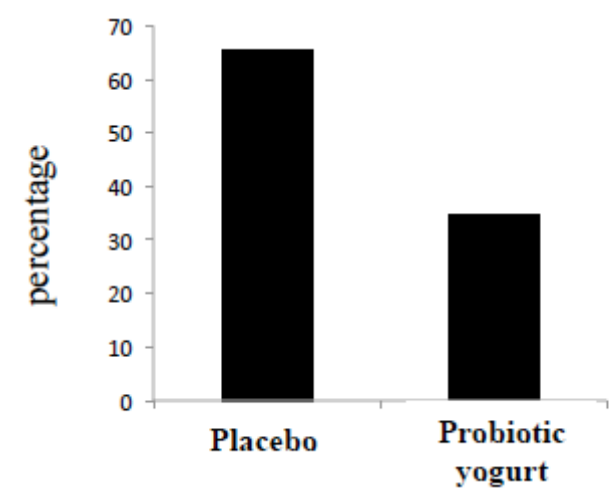

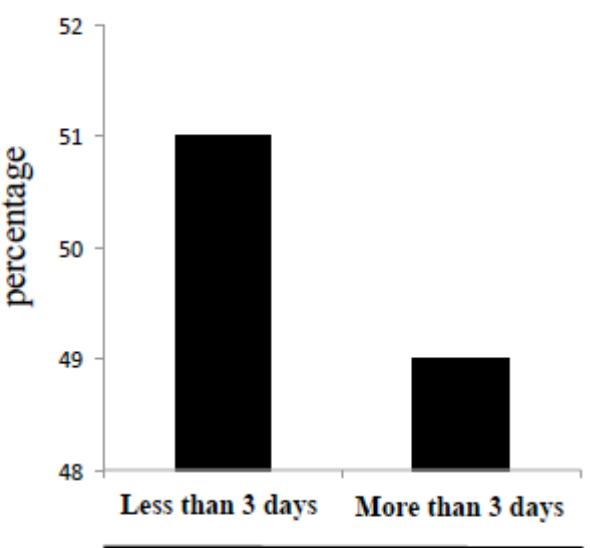

Probiotic yogurt

C

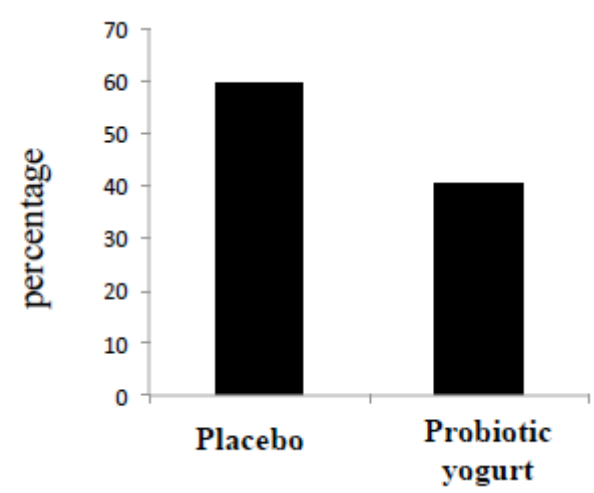

Figure 3: Effects of a probiotic yogurt containing the strain Lactobacillus rhamnosus CRL1505 on the severity of common infectious diseases in pre-school children. (A) Symptom duration, (B) presence of fever, (C) use of antibiotics.

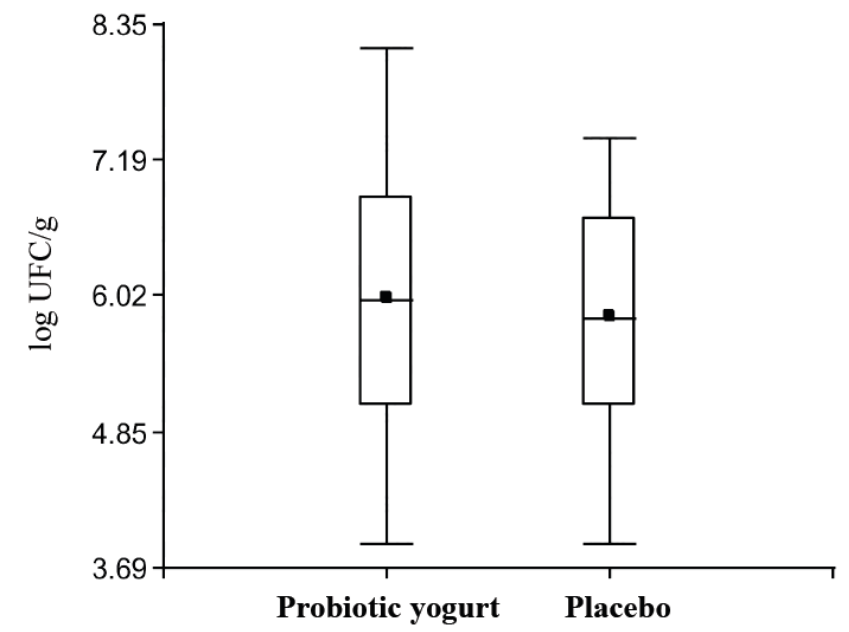

Figure 4: Effects of a probiotic yogurt containing the strain Lactobacillus rhamnosus CRL1505 on faecal concentration of lactic acid bacteria in pre-school children.

\section{Levels of Immunoglobulin A in Saliva}

We performed the determination of $\lg A$ in saliva as a measure of mucosal immunity. Samples were taken the day before the begining of the intervention (basal levels) and at the end of probiotic or placebo administration (post-treatment levels). No significant differences were observed in the basal levels of $\lg \mathrm{A}$ when the probiotic yogurt and the placebo were compared (Figure 5). On the contrary, we observed a significant increase in $\lg A$ levels in children who received the probiotic yogurt while the post-treatment levels of $\lg A$ in the placebo group were similar to those found at the beginning of the trial (Figure 5). Therefore, the consumption of probiotic containing $L$. rhamnosus CRL1505 strain is able to improve mucosal immunity as reveled by the levels of $\lg A$ in saliva.

\section{DISCUSSION}

Reducing the preventable childhood illnesses among preschool children in developing countries is an important public health goal, that would not only impact mortality but would also impact better development of children. It has been estimated that 5.2 million children under five years of age die every year due to preventable infectious diseases like pneumonia and diarrhea [1]. Moreover, recent findings suggest $21 \%$ of 


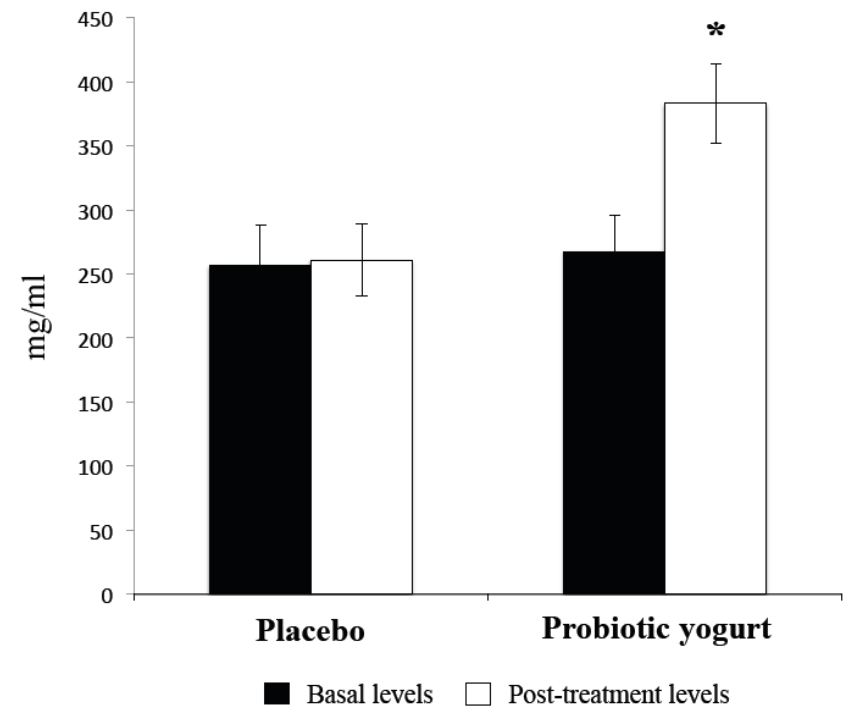

Figure 5: Effects of a probiotic yogurt containing the strain Lactobacillus rhamnosus CRL1505 on saliva IgA levels in pre-school children. (A) basal levels at the beginning of the trial, (B) levels of $\lg A$ after six month of treatment. * Significant differences when compared with the placebo group $(P<0.05)$.

global deaths in children younger than 5 years of age are attributable to malnutrition and its synergistic relationship with preventable infectious diseases [2, 3]. Our region is no exception to these global statistics. Fortification with probiotics may provide one of the potential interventions to reduce the burden of common childhood morbidities [25]. Data on the use of probiotics in preventing common childhood illnesses in a community setting from developing countries is lacking as are data evaluating the effect on illness other than diarrhea. In this study, we report the first randomized controlled trial, evaluating the effect of a probiotic yogurt on both gut and non-gut related illnesses among children from Tucuman, Argentina. Children in probiotic yogurt and placebo groups consumed similar amounts of yogurt, and yogurt in both groups was isoenergic, with identical macronutrient quality and quantity as well as quantity of vitamins and minerals. The only difference was that the yogurt for children allocated to the probiotic group delivered additionally $10^{8} \mathrm{CFU} /$ day of the probiotic strain L. rhamnosus CRL1505.

We demonstrated that administration of $L$. rhamnosus CRL1505 improved mucosal immunity and reduced the incidence and severity of intestinal and respiratory infection in children. When we studied the type of infectious events according to their location and symptoms, the frequency of them was consistent with the prevalence reported in our country. The most common infectious diseases were upper respiratory infections, followed by angina and then lower respiratory infections (acute bronchitis) and diarrhea $[18,21,22,23]$. We registered that $34 \%$ of children who consumed the probiotic yogurt showed some type of infectious event, while in the placebo group this value was higher reaching a $66 \%$ of children. These results demonstrate a significant reduction in occurrence of infectious events associated with consumption of L. rhamnosus CRL1505. We also evaluated the presence or absence of fever during infectious events as well as the need of antibiotic treatment in children who had infections, as indicators of severity. There was a significant decrease in the presence of fever in children who consumed probiotic yogurt as well as a slight decrease in the need for antibiotic treatment, indicating less serious infections in relation to the placebo group.

Several studies have demonstrated that probiotics are able to improve intestinal immunity and reduce susceptibility to intestinal pathogens in children. For example, a study of young children afflicted with rotavirus gastroenteritis showed that $L$. reuteri significantly shortened the duration of diarrhea [26]. Another clinical trail demonstrated that children with mild diarrhea who consumed the combination of $L$. rhamnosus and $L$. reuteri experienced a reduction in the duration of the diarrhea [27]. More recently, a metaanalysis performed by Salari et al., [28] showed that probiotics administration significantly decreased the duration of diarrhea and fever in children. These studies illustrated the beneficial effects of different lactobacilli on gastrointestinal infections and they are in accordance with our results. On the contrary, few studies have evaluated whether probiotics are capable of preventing respiratory infections and reducing their severity in children. Some studies have examined the role of probiotics in the prevention of respiratory tract infections in healthy individuals [29, 30]; particularly those in children's daycare centers [11, 31]. Results from a study evaluating Bifidobacterium lactis or Lactobacillus reuteri versus placebo did not show a beneficial probiotic effect on the rate and duration of respiratory illnesses [31]. On the other hand, randomized, double- blind, placebo-controlled studies performed in daycare centers showed that administration of Lactobacillus $\mathrm{GG}$ resulted in a reduction in the number of children suffering from respiratory tract infections [11, 13]. Moreover, treatment with Lactobacillus GG significantly reduces the risk for developing nosocomial respiratory tract infections in children who were hospitalized on a 
pediatric ward [12]. These results suggest that not all the probiotic strains that are able to stimulate intestinal defenses are capable to improve respiratory immunity. Therefore, certain LAB strains may share certain functional properties. However, some of them can perform a functional role better than others, so it is important to carry out thorough studies on specific strains, according to their therapeutic use. In this sense, our systematic studies in animals on the ability of LAB strains to improve respiratory immunity, allowed us to select a strain that is likely to have a beneficial effect in humans, which has been demonstrated in this clinical trial.

The study of the mechanisms responsible for the beneficial role of probiotics on the gut have documented direct anti-microbial effects and improvement in mucosal barrier function as a result of the effects of probiotics on both innate and adaptive immunity. However, the mechanisms responsible for the improvement of defenses against upper respiratory infections have not been fully elucidated. We have made some progress in the knowledge of the immunological mechanisms involved in the protective effect of L. rhamnosus CRL1505 against respiratory pathogens [32].

Our laboratory has demonstrated that the oral administration of certain $L A B$ strains is able to induce the $\lg A$ cycle and increase the $\lg \mathrm{A}^{+}$cell population in the respiratory tract $[7,33]$. In the case of $L$. rhamnosus CRL1505 we found that this strain was capable of increasing the number of $\lg \mathrm{A}^{+}$cells in intestine and bronchus of mice [15]. We also found that $L$. rhamnosus CRL1505 was able to improve the production of anti-pneumococcal $\lg \mathrm{A}$ in the airways [15]. The production of $\lg A$ in the respiratory tract during an infectious process is important because it prevents colonization of mucosal tissues and subsequent spreading into the systemic circulation [34]. Additionally, $\lg \mathrm{A}$ antibodies can bind antigens and minimize their entry with a consequent reduction in inflammatory reactions, which prevents potentially harmful effects on the tissue. In this study we found improved levels of $\lg A$ in children that received the probiotic, therefore we assume that the stimulation of the $\lg A$ cycle and the improvement of the levels of $\lg A$ induced by the L. rhamnosus CRL1505 could partly explain the greater resistance of children to respiratory infections.

On the other hand, it is known that the symptoms associated with common cold are a result of the inflammatory response by the host towards the infection. Therefore, compounds with the capacity to improve immunity and control unproductive inflammation are supposed to be effective antivirals. In this sense, we have demonstrated in animal studies that treatments with $L$. rhamnosus CRL1505 prior to pneumococcal infection induced a significant increase in IL-10 in lung and serum [15]. Consequently, in agreement with other reports [35], IL-10 was valuable to attenuate inflammatory damage and pathophysiological alterations in lung infected with pneumococci. According to our results, L. rhamnosus CRL1505 treatment would beneficially regulate the balance between pro- and anti-inflammatory cytokines, allowing a more effective inflammatory response against infection. This controlled inflammatory response induced by $L$. rhamnosus $C R L 1505$ could explain the beneficial effect of this strain in the incidence and severity of common cold observed in our study.

Although we did not evaluate etiology of diarrhea in this study, previous evaluations have shown rotavirus and adenovirus to be responsible for most cases of intestinal infection in children [23]. In addition, viral pathogens, such as respiratory syncytial virus, human metapneumovirus, influenza A virus, parainfluenza viruses, and rhinoviruses are considered the major viruses that can cause respiratory tract diseases in children [20]. Therefore, the findings of this study suggest that administration of $L$. rhamnosus CRL1505 may provide one of the potential interventions to reduce the burden of common childhood morbidities, especially those associated to viral infections. The precise cellular and molecular mechanisms involved in the improvement of antiviral immunity induced by $L$. rhamnosus CRL1505 is an interesting topic for future investigations.

In this work, we demonstrated that consumption of a fermented dairy product containing $L$. rhamnosus CRL1505 is associated with a significant decrease in the duration and severity of mucosal infections providing the first evidence that a dairy fermented product containing this immunobiotic strain may have a beneficial effect against respiratory infections in young children. Based on the results summarized in this work and given the high morbidity and mortality in children especially associated with airways infectious diseases, dietary intervention using a dairy product containing the probiotic strain $L$. rhamnosus CRL1505 can be useful to improve health status of this vulnerable population. From the above, this new probiotic strain has been 
included into official National Nutritional Programs in Argentina. Since 2008, the probiotic yogurt containing L. rhamnosus CRL1505 (YOGURITO ${ }^{\circledR}$ ) is given daily to more than 200 thousand children in Tucumán city thanks to the Government actions (Social Development Ministry). The use of the immunobiotic CRL1505 strain to improve children health has already transcended the limits of Tucuman province since other provinces in Argentina are also participating in this Social Program and more children are being benefited with the probiotic YOGURITO ${ }^{\circledR}$.

The social project YOGURITO ${ }^{\circledR}$ has multiple positive impacts at different levels, in the regional economy by encouraging both the local milk production and the competitiveness in small and medium enterprises (SMEs) through innovation and dairy products differentiation; in the children education and health status by improving wellness and the immune system from the basis that "more health is equal to less school desertion".

\section{REFERENCES}

[1] Bryce J, Boschi-Pinto C, Shibuya K, Black RE. WHO Child Health Epidemiology Reference Group. WHO estimates of the causes of death in children. Lancet 2005; 365: 1147-52. http://dx.doi.org/10.1016/S0140-6736(05)71877-8

[2] Lopez AD, Mathers CD, Ezzati M, Jamison DT, Murray CJL. Global and regional burden of disease and risk factors: systematic analysis of population health data. Lancet 2006; 367: 1747-57. http://dx.doi.org/10.1016/S0140-6736(06)68770-9

[3] Black RE, Allen LH, Bhutta ZA, et al. Maternal and child undernutrition: global and regional exposures and health consequences. Lancet 2008; 371: 243-60.

http://dx.doi.org/10.1016/S0140-6736(07)61690-0

[4] Pang IK, Iwasaki A. Control of antiviral immunity by pattern recognition and the microbiome. Immunol Rev 2012; 245: 209-26.

http://dx.doi.org/10.1111/j.1600-065X.2011.01073.x

[5] Villena J, Oliveira ML, Ferreira P, Salva S, Alvarez S. Lactic acid bacteria in the prevention of pneumococcal respiratory infection: future opportunities and challenges. Int Immunopharmacol 2011; 11: 1633-45.

http://dx.doi.org/10.1016/j.intimp.2011.06.004

[6] Villena J, Racedo S, Agüero G, et al. Lactobacillus casei improves resistance to pneumococcal respiratory infection in malnourished mice. J Nutr 2005; 135: 1462-9.

[7] Racedo S, Villena J, Medina M, et al. Lactobacillus casei administration reduces lung injuries in a Streptococcus pneumoniae infection in mice. Microbes Infect 2006; 8: 235966.

http://dx.doi.org/10.1016/j.micinf.2006.04.022

[8] Alvarez S, Herrero C, Bru E, Perdigon G. Effect of Lactobacillus casei and yogurt administration on prevention of Pseudomonas aeruginosa infection in young mice. J Food Prot 2001; 64: 1768-74.

[9] Hori T, Kiyoshima J, Shida K, Yasui H. Augmentation of cellular immunity and reduction of influenza virus titer in aged mice fed Lactobacillus casei strain Shirota. Clin Diagn Lab Immunol 2002; 9: 105-8.

http://dx.doi.org/10.1128/CDLI.9.1.105-108.2002
[10] Morrow LE, Kollef MH, Casale TB. Probiotic prophylaxis of ventilator-associated pneumonia: a blinded, randomized, controlled trial. Am J Respir Crit Care Med 2010; 182: 105864.

http://dx.doi.org/10.1164/rccm.200912-18530C

[11] Hatakka K, Savilahti E, Pönkä A, et al. Effect of long term consumption of probiotic milk on infections in children attending day care centres: double blind, randomised trial. BMJ 2001; 322: 1327.

http://dx.doi.org/10.1136/bmj.322.7298.1327

[12] Hojsak I, Abdović S, Szajewska H, et al. Lactobacillus GG in the prevention of nosocomial gastrointestinal and respiratory tract infections. Pediatrics 2010; 125: e1171-7.

http://dx.doi.org/10.1542/peds.2009-2568

[13] Hojsak I, Snovak N, Abdović S, et al. Lactobacillus GG in the prevention of gastrointestinal and respiratory tract infections in children who attend day care centers: a randomized, double-blind, placebo-controlled trial. Clin Nutr 2010; 29: 312-6.

http://dx.doi.org/doi:10.1038/ejcn.2012.62

[14] de Vrese $M$, Winkler $P$, Rautenberg $P$, et al. Probiotic bacteria reduced duration and severity but not the incidence of common cold episodes in a double blind, randomized, controlled trial. Vaccine 2006; 24: 6670-4.

http://dx.doi.org/10.1016/j.vaccine.2006.05.048

[15] Salva S, Villena J, Alvarez S. Diferential immunomodulatory activity of Lactobacillus rhamnosus strains isolated from goat milk: impact on intestinal and respiratory infections. Int $\mathrm{J}$ Food Microbiol 2010; 141: 82-9.

http://dx.doi.org/10.1016/j.ijfoodmicro.2010.03.013

[16] Salva S, Nuñez M, Villena J, et al. Development of a fermented goats' milk containing Lactobacillus rhamnosus: in vivo study of health benefits. J Sci Food Agric 2011; 91: 2355-62.

http://dx.doi.org/10.1002/jsfa.4467

[17] Salva S, Merino MC, Agüero G, Gruppi A, Alvarez S. Dietary supplementation with probiotics improves hematopoiesis in malnourished mice. PLoS One 2012; 7: e31171. http://dx.doi.org/10.1371/journal.pone.0031171

[18] Bardach A, Ciapponi A, Garcia-Marti S, et al. Epidemiology of acute otitis media in children of Latin America and the Caribbean: a systematic review and meta-analysis. Int $\mathrm{J}$ Pediatr Otorhinolaryngol 2011; 75: 1062-70. http://dx.doi.org/10.1016/j.ijporl.2011.05.014

[19] Rocco R. Periodic fever, aphthous stomatitis, pharyngitis and adenitis: PFAPA syndrome in Argentina. An Pediatr 2011; 74: 161-7. http://dialnet.unirioja.es/servlet/articulo?codigo=3619768

[20] Wolf DG, Greenberg D, Kalkstein D, et al. Comparison of human metapneumovirus, respiratory syncytial virus and influenza A virus lower respiratory tract infections in hospitalized young children. Pediatr Infect Dis J 2006; 25: 320-4.

http://dx.doi.org/10.1097/01.inf.0000207395.80657.cf

[21] Gentile A, Bardach A, Ciapponi A, et al. Epidemiology of community-acquired pneumonia in children of Latin America and the Caribbean: a systematic review and meta-analysis. Int J Infect Dis 2012; 16: e5-15.

http://dx.doi.org/10.1016/j.ijid.2011.09.013

[22] Edmond K, Scott S, Korczak V, et al. Long term sequelae from childhood pneumonia; systematic review and metaanalysis. PLoS One 2012; 7: e31239.

http://dx.doi.org/10.1371/journal.pone.0031239

[23] Stupka JA, Carvalho P, Amarilla AA, et al. National Rotavirus Surveillance in Argentina: high incidence of G9P[8] strains and detection of G4P[6] strains with porcine characteristics. Infect Genet Evol 2009; 9: 1225-31.

http://dx.doi.org/10.1016/j.meegid.2009.07.002 
[24] Empinotti JC, Uyeda H, Ruaro RT, Galhardo AP, Bonatto DC. Pyodermitis. An Bras Dermatol 2012; 87: 277-84. http://dx.doi.org/10.1590/S0365-05962012000200013

[25] Sazawal S, Dhingra U, Hiremath G, et al. Prebiotic and probiotic fortified milk in prevention of morbidities among children: community-based, randomized, double-blind, controlled trial. PLoS ONE 2010; 5: e12164.

http://dx.doi.org/10.1371/journal.pone.0012164

[26] Shornikova AV, Casas IA, Mykkanen H, Salo E, Vesikari T. Bacterotherapy with Lactobacillus reuteri in rotavirus gastroenteritis. Pediatr Infect Dis 1997; 16: 1103-7. http://dx.doi.org/10.1097/00006454-199712000-00002

[27] Rosenfeldt V, Michaelsen KF, Jakobsen M, et al. Effect of probiotic Lactobacillus strains on acute diarrhea in a cohort of nonhospitalized children attending day-care centers. Pediatr Infect Dis J 2002; 21: 417-9. http://dx.doi.org/10.1097/00006454-200205000-00013

[28] Salari P, Nikfar S, Abdollahi M. A meta-analysis and systematic review on the effect of probiotics in acute diarrhea. Inflamm Allergy Drug Targets 2012; 11:3-14.

[29] de Vrese $M$, Winkler $P$, Rautenberg $P$, et al. Effect of Lactobacillus gasseri PA 16/8, Bifidobacterium longum SP $07 / 3$, B. bifidum MF $20 / 5$ on common cold episodes: a double blind, randomized, controlled trial. Clin Nutr 2005; 24: 481-91.

http://dx.doi.org/10.1016/j.clnu.2005.02.006
[30] Kukkonen K, Savilahti E, Haahtela T, et al. Long-term safety and impact on infection rates of postnatal probiotic and prebiotic (synbiotic) treatment: randomized, double-blind, placebo-controlled trial. Pediatrics 2008; 122: 8-12. http://dx.doi.org/10.1542/peds.2007-1192

[31] Weizman Z, Asli G, Alsheikh A. Effect of a probiotic infant formula on infections in child care centers: comparison of two probiotic agents. Pediatrics 2005; 115: 5-9. http://dx.doi.org/10.1542/peds.2004-1815

[32] Villena J, Salva S, Núñez $M$, et al. Beneficial lactobacilli for improving respiratory defenses: the case of Lactobacillus rhamnosus CRL1505. 2012. In: Lactobacillus: classification, uses and health implications. NOVA Science publishers, In press.

[33] Villena J, Medina M, Vintiñi E, Alvarez S. Stimulation of respiratory immunity by oral administration of Lactococcus lactis. Can J Microbiol 2008; 54: 630-8. http://dx.doi.org/10.1139/W08-077

[34] Twigg HL. Humoral immune defense (antibodies): recent advances. Proc Am Thorac Soc 2005; 2: 417-21. http://dx.doi.org/10.1513/pats.200508-089JS

[35] Kerr AR, Irvine JJ, Search JJ, et al. Role of inflammatory mediators in resistance and susceptibility to pneumococcal infection. Infect Immun 2002; 70: 1547-57. http://dx.doi.org/10.1128/IAl.70.3.1547-1557.2002

\section{DOI: http://dx.doi.org/10.6000/1927-3037/2012.01.03.05}

(C) 2012 Villena et al.; Licensee Lifescience Global.

This is an open access article licensed under the terms of the Creative Commons Attribution Non-Commercial License (http://creativecommons.org/licenses/by-nc/3.0/) which permits unrestricted, non-commercial use, distribution and reproduction in any medium, provided the work is properly cited. 